\title{
Review of: "A Novel Model for Encephalomyosynangiosis Surgery after Middle Cerebral Artery Occlusion-Induced Stroke in Mice"
}

\author{
Zhijun Zhang ${ }^{1}$ \\ 1 Shanghai Jiaotong University
}

Potential competing interests: The author(s) declared that no potential competing interests exist.

The authors use encephalomyosynangiosis surgery (EMS) to improve angiogenesis after ischemic stroke, the results showed that graft musle tissue remained viable 21days after surgery. it is novel treatment for angiogenesis after stroke. However, EMS is performed 3-4 hours after MCAO, the time window is very short, which has no advantage compared to tissue plasminogen activator (tPA) treatment after stroke. On the other hand, the authors only measure the graft tissue viability, angiogenesis in peri-infarct area is also needed to detect, which is the purpose of EMS. 